\title{
Molecular dynamics on a realistic model for a strong glass
}

\author{
R. Fernández-Perea and F. J. Bermejo \\ Instituto de Estructura de la Materia, Consejo Superior de Investigaciones Científicas, Serrano 123, E-28006 Madrid, Spain \\ E. Enciso \\ Departamento de Química-Física I, Universidad Complutense de Madrid, E-28040 Madrid, Spain
}

(Received 27 July 1995; revised manuscript received 4 October 1995)

\begin{abstract}
Thermal motions in a microscopic model for a strong glass $\left(\mathrm{B}_{2} \mathrm{O}_{3}\right)$ are studied by means of computer molecular dynamics simulations. A decomposition of the atomic dynamics in terms of normal modes allows the separation of the truly harmonic components of the generalized frequency distributions. This enables us to discuss a number of magnitudes relevant to current issues on glassy dynamics on quantitative grounds. In particular, the microscopic origin of quantities such as the atomic mean-square displacements, the characterization of mode eigenvectors associated with well-defined spectral features, or the origin of the low-frequency peak appearing in the $S(Q, \omega)$ dynamic structure factor, are analyzed in some detail.
\end{abstract}

\section{INTRODUCTION}

The quantitative understanding of the complicated atomic dynamics exhibited by supercooled liquids as well as that characteristic of the glassy state achieved upon crossing from above the glass transition still constitutes a challenge to efforts within a varied set of subspecialities within condensed matter sciences. One of the main difficulties regards the coexistence within comparable time scales of motions representing disparate phenomena which can hardly be separated, although attempts in this direction have recently appeared. ${ }^{1,2}$ There, the existence of well-differentiated motions is postulated, ascribing as "relaxations" those taking place within meso- and macroscopic scales of time and length, whereas those occurring within the picosecond scale are termed as "vibrations." The experimental evidence upon which such a distinction is substantiated heavily relies upon some model response function which is used to separate the highfrequency vibrational component from the low-frequency spectrum. Because of the complicated dynamics exhibited by most materials being scrutinized following such approaches, most model response functions regard oversimplified (Debye-like) representations of the vibrational frequency distributions, ${ }^{1}$ the validity of which can only be justified by the complicated nature of the materials under consideration.

Our aim here is to illustrate an alternative way to unravel the microscopic origin of the different frequency regions in the dynamic response of disordered matter without making recourse to such simplifying assumptions. The present efforts are focused onto the description of the atomic dynamics of a strong glass which is carried after its decomposition into "normal modes," which are calculated for a relevant (but large) atomic cluster. As shown below, such a separation enables us to discuss the microscopic origin of some characteristic fingerprints of glassy dynamics such as the large atomic mean-square displacements on a quantitative basis. On the other hand, such an approach provides a truly microscopic correlate for some aspects currently discussed in terms of phenomenological constructs such as the "soft-mode" concept $^{3}$ a tool recently employed in discussions on glassy dynamics.

The present results will also try to contribute towards the clarification of the origin of the inelastic intensities at frequencies of about $0.5-10 \mathrm{meV}$ which give rise to a finitefrequency feature in the neutron and Raman spectra commonly referred to as the "Boson" peak, ${ }^{4}$ and which is considered to be one of the universal fingerprints of the glassy dynamics. For such a purpose, the advantage of the normal-mode decomposition of the atomic dynamics will be fully exploited since it provides valuable information regarding the atomic mode eigenvectors and, therefore, enables the characterization of the excitations sampled at frequencies comparable with those of such a peak. Additional evidence regarding the origin of such a peak will, on the other hand, follow from a detailed study of the wave-vector dependence of the inelastic intensity up to length scales close to those characteristic of full hydrodynamic behavior. The implications of the present results regarding current issues on glassy dynamics will finally be discussed.

\section{COMPUTATIONAL DETAILS}

A substantial number of papers regarding the microscopic modeling of vibrations in glassy boron trioxide have appeared. ${ }^{5}$ Their degree of sophistication goes from the relatively simple ball and stick models used by Bell et al. ${ }^{5}$ or the central force network dynamics of Galeener and Thorpe, ${ }^{5}$ to those employing calculations on Bethe lattices of Barrio, Castillo-Alvarado, and Galeener. ${ }^{5}$ By construction, such efforts only regard vibrations at relatively high frequencies (above some $5 \mathrm{meV}$ ) which appear in the Raman spectra as well-resolved features, ${ }^{5,6}$ whereas our main interests are focused on the low-frequency dynamics. For such a purpose, we have chosen one of the available empirical potential models which has been developed ${ }^{7}$ to account for the most salient features of the structure ${ }^{8}$ and thermodynamics ${ }^{9}$ of the material, and considers all the atomic motions on equal grounds.

The computer simulations were carried out by means of NVT molecular dynamics (i.e., constant number of particles, volume, and temperature) using as a starting point the fourth 
TABLE I. Finite-size effects upon the frequency moments of the spectrum of eigenvalues of the dynamical matrix for samples of 420 and 1500 particles. The last column gives the relative error.

\begin{tabular}{lccc}
\hline \hline$\left\langle\omega^{n}\right\rangle$ & $N=420$ & $N=1500$ & Error \\
\hline$n=4$ & $2.16583 \times 10^{8}$ & $2.18640 \times 10^{8}$ & $0.941 \%$ \\
$n=3$ & 1434521. & 1440889. & $0.442 \%$ \\
$n=2$ & 10383.55 & 10390.18 & $0.064 \%$ \\
$n=1$ & 86.08042 & 85.85067 & $0.268 \%$ \\
$n=0$ & 1. & 1. & $0.0 \%$ \\
$n=-1$ & 0.016283 & 0.015104 & $7.809 \%$ \\
$n=-2$ & 0.003913 & 0.002190 & $78.65 \%$ \\
$n=-3$ & 0.002212 & 0.000827 & $167.5 \%$ \\
\hline \hline
\end{tabular}

potential given in Table I of Ref. 7 which includes two(Born-Mayer-Huggins) and three-body (bond-bending) interactions. The potential parameters were then refined by comparison of the experimental $S(Q)$ static structure factor ${ }^{10}$ measured by neutron diffraction with the calculated magnitude. In such a way, refined estimates for the equilibrium value of the $\mathrm{B}-\mathrm{O}-\mathrm{B}$ bond angle of $\theta_{0}=110^{\circ}$, potential parameters for boron-boron interactions of $A_{\mathrm{BB}}=5.96 \times 10^{3} \mathrm{~kJ}$ $\mathrm{mol}^{-1}$, and $A_{\mathrm{OO}}=75.65 \times 10^{3} \mathrm{~kJ} \mathrm{~mol}^{-1}$ for oxygen-oxygen were derived. A comparison of the calculated structure factor with the experimental curve is shown in Fig. 1. In general terms, the agreement between experiment and simulation is deemed as acceptable, even if some drastic mismatch between calculation and measurement occurs about the first diffraction peak located at a momentum transfer of $Q \approx 1.58 \AA^{-1}$. Such a discrepancy arises from the fact that, as shown in the inset of Fig. 1, the three partial structure factors show narrow peaks within that region of momentum transfers so that perfect matching of the three are required to reproduce the width and intensity of the experimental pattern. Up to the present moment we have been unable to find a more reliable parameter set since improvements in the fit of the first peak resulted in a strong mismatch of subsequent oscillations. Most of the results here refer to a simulation regarding 420 atoms and a density of $1.82 \mathrm{~g} \mathrm{~cm}^{-3}$. Samples at $T=300 \mathrm{~K}$ were prepared following Ref. 7. Lowtemperature $(0.01 \mathrm{~K})$ runs were prepared by scaling the temperature down to $T=0.1 \mathrm{~K}$ during $5 \mathrm{ps}$, and subsequent cooling for the next 3 ps down to the sought temperature. A test of the mechanical stability of the system was carried out by means of computation of the average force on an atom which turned out to be of the order of $10^{-14} \mathrm{~N}$, a quantity to be compared with values of $10^{-6} \mathrm{~N}$ of the force constants.

Finite-size effects were explored by means of comparison of results from simulations using 420 and 1500 atoms. A rather small size dependence was found as shown by results given in Table I. From there it is seen that the main finite-size effects are concentrated on a narrow range of frequencies $\omega$ about 0 . Notice however that the large differences regarding the negative moments mostly arise as a consequence of the divergent behavior of the $\omega^{-n}$ terms in the integrand which lead to large statistical errors.

To approach the hydrodynamic regime, calculations for the $S(Q, \omega)$ dynamic structure factor were carried out using a large simulation box $174.74 \AA \times 17.47 \AA \times 17.47 \AA$ containing 4200 atoms. This enables the exploration of correlations taking place at scales up to approximately $180 \AA$ of length and times of 16.4 ps.

Although a normal-mode description seems the most natural starting point to enable a quantitative study of glassy dynamics, a number of difficulties mostly regarding the quality of the available data have hindered any substantial progress in this direction. As a matter of fact, specific schemes to derive information of this kind from the measured $S(Q, \omega)$ dynamic structure factor were proposed some time ago, ${ }^{11}$ and results of an application to the study of the dynamics of a Lennard-Jones glass were reported by Rahman, Mandell, and McTague ${ }^{12}$ almost two decades ago. A reawakening of the interest in this topic has been witnessed in recent times motivated by efforts to describe the collective dynamics of liquids within such a framework. ${ }^{12}$ From there,

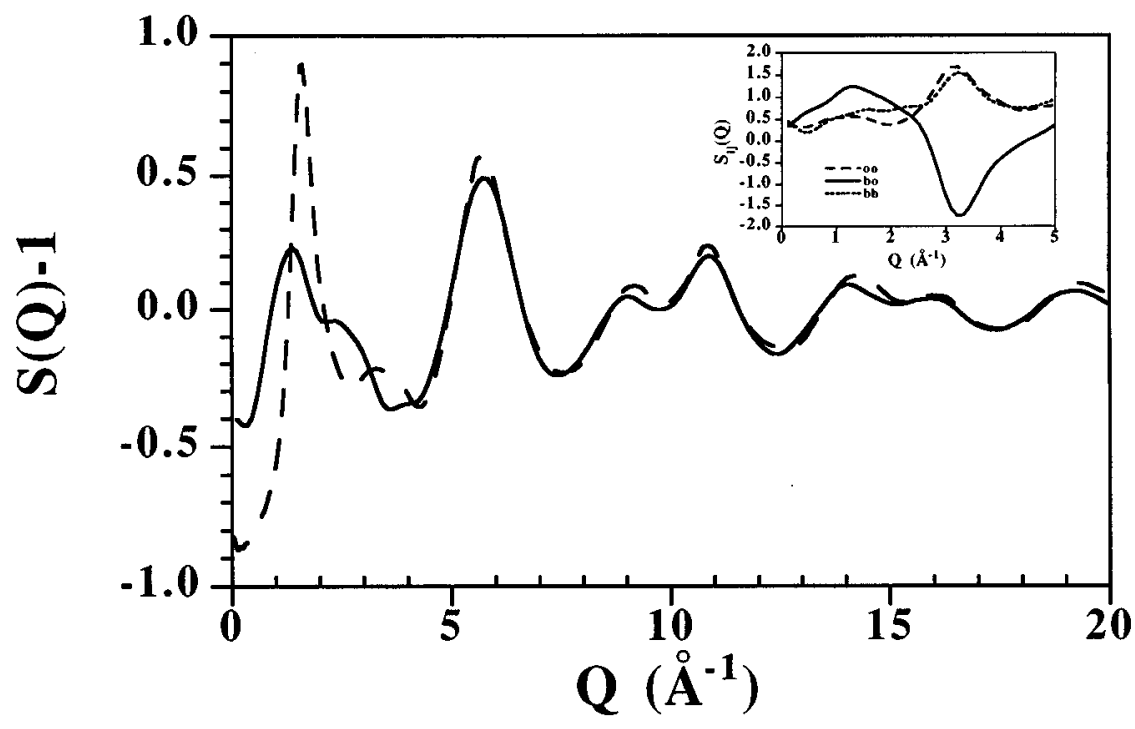

FIG. 1. A comparison between the $S(Q)-1$ static structure factor as reported by Johnson, Wright, and Sinclair (Ref. 10) (dashed line) and the one resulting from the present calculation (solid line). 
two different routes have been proposed ${ }^{13}$ for the description of the harmonic dynamics in terms of discrete modes. Both of them start from consideration of a given atomic configuration $\mathbf{R}_{0}$ chosen from the classical trajectory of a system of $N$ particles of mass $M$ interacting through a $\Phi(\mathbf{R})$ potential, and follow an expansion,

$$
\begin{gathered}
\Phi(\mathbf{R})=\Phi\left(\mathbf{R}_{0}\right)-\mathbf{F} \cdot\left(\mathbf{R}-\mathbf{R}_{0}\right) \\
+\frac{1}{2}\left(\mathbf{R}-\mathbf{R}_{0}\right) \cdot \mathbf{D} \cdot\left(\mathbf{R}-\mathbf{R}_{0}\right)+\cdots \\
F_{i j}=\left.\frac{\partial \Phi(\mathbf{R})}{\partial R_{i j}}\right|_{\mathbf{R}=\mathbf{R}_{0}} ; \quad D_{i j, k l}=\left.\frac{\partial^{2} \Phi(\mathbf{R})}{\partial R_{i j} \partial R_{k l}}\right|_{\mathbf{R}=\mathbf{R}_{0}},
\end{gathered}
$$

where $\mathbf{F}$ and $\mathbf{D}$ stand for the force vector and dynamical matrix, respectively. A description in terms of instantaneous modes (INM) would then correspond to that where configurations are chosen from the glass trajectory, the mode frequencies being determined by the $\lambda_{i}=M \omega_{i}^{2}(\mathbf{R})$ eigenvalues of $\mathbf{D}$ and the frequency distribution (i.e., vibrational density of states) calculated from

$$
Z(\omega) \equiv\left\langle\frac{1}{3 N} \sum_{i=1}^{3 N} \delta\left(\omega-\omega_{i}\right)\right\rangle_{\mathrm{Av}},
$$

where the index on the angle bracket stands for an average over different configurations. Because the sample obtained by quenching a high-temperature liquid will contain some regions of significant strain, this will make the force term to assume finite values which in turn gives rise to mode instabilities as attested by imaginary values of $\omega_{i}$. Rather than being a nuisance, these frequencies which correspond to unstable motions can provide valuable information regarding some thermostatic properties such as the average potential energy and constant-volume heat capacity, ${ }^{14}$ and therefore serve to quantify the dynamic state of a finite-temperature system. On the other hand, an alternative approach could be pursued where diagonalization of the Hessian matrix is only done after performing a number of steepest descents to allow partial relaxation of unstable modes. In consequence, the modes calculated in such a way, usually referred to as quenched normal modes (QNM) being restricted to potential local minima do not show mode instabilities, and therefore the calculated frequency distributions will be devoid of a lobe at imaginary frequencies. A comparison between results obtained following the two approaches also shows that the QNM distributions evidence a more structured shape than the ones calculated from INM analyses (as an example see Fig. 2 of Ref. 13). The main advantage in the use of a QNM description perhaps regards its usefulness as a source of information for studies on transitions between different stable configurations of a liquid ("inherent liquid structures"15).

\section{RESULTS AND DISCUSSION}

\section{A. Frequency distributions and thermodynamics}

The experimental ${ }^{6} Z(\omega)$ distribution for relatively low energy transfers and the one calculated from the time Fourier transform of the atomic velocity autocorrelation function, are compared in Fig. 2. As shown there, the simulation gives an

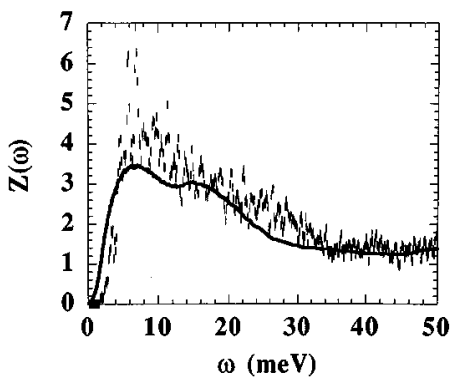

FIG. 2. A comparison between the calculated (vertical bars) and measured (thick solid line) frequency distributions for energy transfers below $50 \mathrm{meV}$. Both curves have been normalized to unit area to facilitate comparison. Experimental measurement corresponds to $T=100 \mathrm{~K}$.

acceptable account of the experimental function, even though the features giving rise to the first peak at approximately $5 \mathrm{meV}$ are somewhat overemphasized in the calculation and the leading edge of the calculated $Z(\omega)$ is shifted by some $0.8 \mathrm{meV}$ with respect to experiment.

The distributions spanning the whole range of frequencies of interest and corresponding to temperatures of 0.01 and $300 \mathrm{~K}$ are shown in Fig. 3. The inset of Fig. 3(a) is to be compared with experimental neutron spectra shown in Fig. 3 of Ref. 16 which displays a relatively broad feature at 82 $\mathrm{meV}$ and two relatively narrower ones (width of about 2 $\mathrm{meV}$ ) at $\approx 90$ and $100 \mathrm{meV}$. The calculation shows some features clearly related to those seen by experiment, although some mismatch in frequencies is to be noted. In particular, the band at $82 \mathrm{meV}$ (labeled as 4 in the Galeener and Thorpe nomenclature ${ }^{5}$ ) appears in the calculation downshifted by some $8 \mathrm{meV}$, that of $\approx 90 \mathrm{meV}$ (labeled as 5 in the figure) correlates with intensities of about $88 \mathrm{meV}$ and that of 100 $\mathrm{meV}$ has a clear counterpart in a sharp peak at about the same frequency. The last feature appears in the calculation with an intensity substantially reduced with respect to experiment, and its origin still is a matter of debate. Some authors, ${ }^{6,16}$ ascribe its origin to a breathing mode of the $\mathrm{B}_{3} \mathrm{O}_{6}$ boroxol ring, whereas results from calculations of $\mathrm{B}_{3} \mathrm{O}_{6}$ rings on a Bethe lattice carried out by Barrio, CastilloAlvarado, and Galeener ${ }^{5}$ seem to contradict such an assignment, since such a mode was found to correspond to a far less intense feature appearing in the horizontally polarized Raman spectrum at $\approx 75 \mathrm{meV}$. On the other hand, and since no measurable amount of boroxol rings is present in the simulation box, it seems clear that the relatively small feature seen here at $\approx 100 \mathrm{meV}$ cannot be assigned to such a motion, and further discussion regarding the origin of the most prominent spectral features is deferred to the next section where these topics are discussed in some more detail.

The harmonic frequency distributions as calculated from INM analysis using Eq. (2) are also compared with those calculated from the atomic velocity autocorrelations in Fig. 3. As expected, the harmonic distributions show a rather weak dependence with temperature, being the small differences between both temperatures confined to a region about $80 \mathrm{meV}$ where the high-temperature system shows a smoother behavior, and also to the lobe of imaginary frequencies which, in contrast with findings regarding simpler systems, ${ }^{12,17}$ shows a far milder dependence with tempera- 

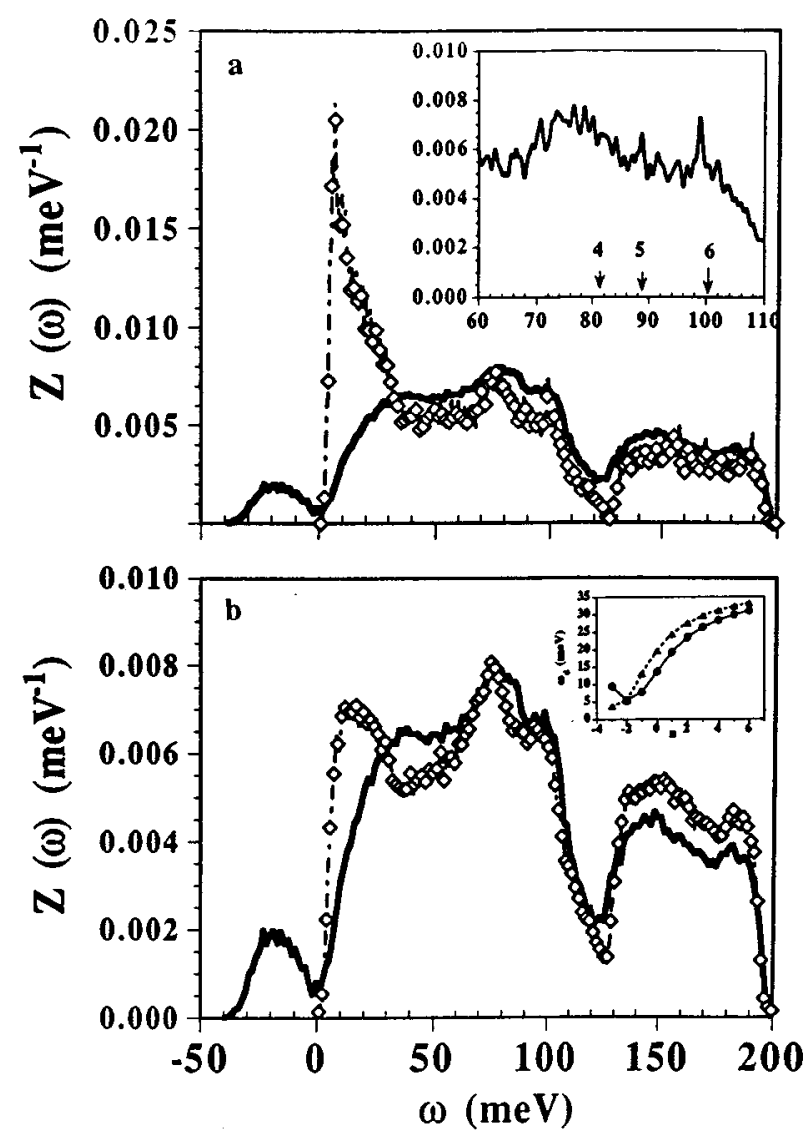

FIG. 3. Generalized frequency distributions as calculated from the atomic velocity autocorrelation functions (lozenges and dashdot lines) and those derived from decomposition of the atomic dynamics into INM (solid lines). The upper frame shows the two quantities for $T=0.1 \mathrm{~K}$. The inset shows an enlargement of part of the quantity calculated from the atomic velocity autocorrelation. Digits and arrows show the approximate location of peaks in the Raman spectra and follow the nomenclature of Galeener and Thorpe. The lower frame shows data for $T=300 \mathrm{~K}$. The inset shows the reduced frequency moments $\left\langle\omega_{D}^{n}\right\rangle$ (triangles for $T=300 \mathrm{~K}$, circles for $T=0.1 \mathrm{~K}$ ).

ture. In opposition, the spectra calculated from the atomic velocity correlations, which include all possible sources of anharmonicity, show a far more dramatic dependence with temperature, especially for frequencies below some $20 \mathrm{meV}$, which is strongly enhanced at low temperatures. Such a counterintuitive result (i.e., an increase in intensity at low frequencies is common at temperatures close to $T_{g}$ due to the onset of quasielastic scattering) has a clear experimental counterpart since available neutron scattering data have also unveiled such an effect. ${ }^{18}$ For frequencies $40 \mathrm{meV}$ $\leqslant \omega \leqslant 130 \mathrm{meV}$, the two $Z(\omega)$ curves just referred can be scaled to a common one, something which serves to set a frequency domain where vibrations show a harmonic behavior. Such a scaling cannot be followed to higher frequencies since vibrations above such a limit mostly involve the motion of boron atoms which, due to its relatively light mass exhibit substantial anharmonic effects. On quantitative grounds the relative importance of the anharmonic effects is best specified in terms of the reduced frequency moments, $\left\langle\omega^{n}\right\rangle=\left\{\frac{1}{3}\left[3+\int d \omega \omega^{n} Z(\omega)\right]\right\}^{1 / n}$ which are also shown in the

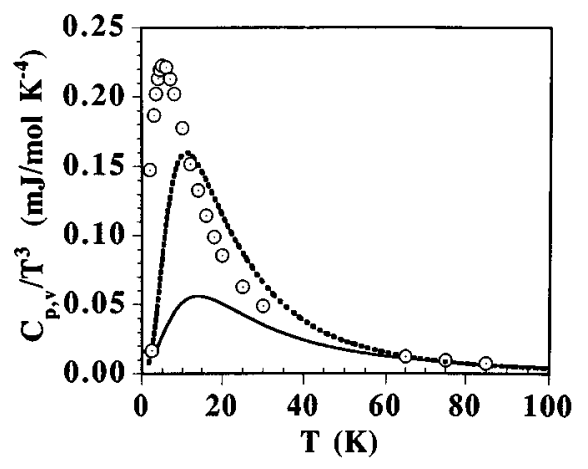

FIG. 4. $C / T^{3}$ heat-capacity data of White, Collocott, and Cook (Ref. 9) (circles) and that calculated from the $Z(\omega)$ at $0.1 \mathrm{~K}$ (dashes) and $300 \mathrm{~K}$ (solid).

figure. All the spectral moments corresponding to the hightemperature calculation are above those regarding $T=0.1 \mathrm{~K}$, and this translates into different values for several thermodynamic properties which can be calculated from these magnitudes. In particular, the limiting values of the Debye temperature concerning the heat capacity and entropy for high temperatures result as $\Theta_{\infty}^{C}=\hbar \omega_{D}(2) / k_{B}=318 \mathrm{~K}$ and $\Theta_{\infty}^{S}=\hbar \omega_{D}(0) / k_{B}=227 \mathrm{~K}$ with $\omega_{D}(n)=\left[(n+3)\left\langle\omega^{n}\right\rangle / 3\right]^{1 / n}$. Such values which correspond to the simulation performed at $300 \mathrm{~K}$ can be compared with the experimental Debye temperature of $259 \mathrm{~K}$ (Ref. 19) derived from low-temperature specific-heat data. A better agreement is found if data of 0.01 $\mathrm{K}$ are considered instead since a value of $\Theta_{\infty}^{C}=271 \mathrm{~K}$ would then result. A comparison of both values serves then to set some bounds to the contribution of anharmonic processes to the measured heat capacity and entropy, which, according to this, should be large but not exceeding some $15 \%$ of the experimental heat capacity at $300 \mathrm{~K}$.

The harmonic heat capacity $C_{v}(T)$ evaluated from $Z(\omega)$ for $T=300 \mathrm{~K}$ yields a value of $12.01 \mathrm{~J} \mathrm{~mol}^{-1} \mathrm{~K}^{-1}$, to be compared with an experimental one of $12.31 \mathrm{~J} \mathrm{~mol}^{-1}$ $\mathrm{K}^{-1} \cdot{ }^{9}$ At low temperature $(10 \mathrm{~K})$ our result gives $0.16 \mu$ $\mathrm{J} \mathrm{mol}^{-1} \mathrm{~K}^{-4}$, which compares well with that measured by Ramos et al. $^{9}$ once the linear contribution arising from twolevel states is accounted for. The maximum of the calculated $C(T) / T^{3}$ curve is located about $8 \mathrm{~K}$, that is upshifted some 2-3 K with respect to experiment, a fact also evidenced by comparison with the data of White, Collocott, and Cook ${ }^{9}$ shown in Fig. 4.

Quantitatively, the extent of anharmonic interactions (neglecting thermal expansion effects) can be estimated from the temperature dependence of the frequency moments. In particular, their contribution to the thermodynamic functions is accounted by means of a quasiharmonic approximation following Hui and Allen, ${ }^{20}$ where the entropy and a firstorder correction to the high-temperature heat capacity are evaluated as

$$
\begin{gathered}
S(T)=3 R \int d \omega Z(\omega, T)[-\ln (2 \sinh x)+x \operatorname{coth} x] \\
\Delta C_{p}=-3 R \frac{\partial \int d \omega Z(\omega, T) \ln \omega}{\partial \ln T}
\end{gathered}
$$




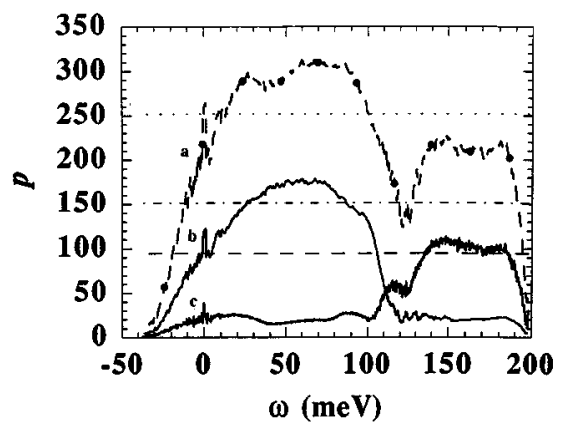

FIG. 5. Mode participation ratios as defined by Eq. (3). Line a shows the total (averaged over all atoms) quantity and is normalized to the total number of atoms within the simulation cell. Modes above the dotted line are above some $p \geqslant 0.6$ and are to be considered as fully extended. Lines $b$ and $c$ display the quantities (unnormalized) for oxygen and boron atoms. The magnitude of the function regarding the cross terms can be inferred by difference with the total ratio. The extended modes for $\mathrm{O}$ atoms would correspond to those above the horizontal dash-dotted line (at $p=151)$ and those involving extended motions of borons would be those above $p=101$ shown by the dotted line.

with $x=\hbar \omega / k_{B} T$, and $Z(\omega, T)$ stand for the temperaturedependent frequency distributions. Evaluation of $\Delta C_{p}$ from the calculated distributions gives a correction which represents a lowering of the heat capacity with respect to its harmonic value at $T=300 \mathrm{~K}$ by $1.2 \%$. On the other hand, rather more drastic effects are noticeable in the entropies calculated within the quasiharmonic approximation. ${ }^{20}$ The calculated value for this function yields at $300 \mathrm{~K} 10.58 \mathrm{~J} \mathrm{~mol}^{-1}$ $\mathrm{K}^{-1}$, which is about $30 \%$ below the value that would be obtained for a harmonic system characterized by the frequency distribution corresponding to $0.1 \mathrm{~K}$.

The estimation of the atomic mean-square displacements was carried first within the (quasi-)harmonic approximation from integrals over the $Z(\omega)$ distributions. A value of 0.017 $\AA^{2}$ was found for the $T=0.01 \mathrm{~K}$ case which is to be compared with a lower bound for this property of some 0.006 $\AA^{2}$ derived from diffraction studies, ${ }^{8}$ whereas a value of $0.096 \AA^{2}$ is calculated for the high temperature.

\section{B. Dynamic correlations at microscopic scales}

From the ,D, dynamical matrix (1), information regarding the geometry of the atomic motions becomes available in terms of the $\gamma_{m, i}$ mode eigenvectors which are normalized as $\Sigma_{i, m}\left(\gamma_{m, i}\right)^{2}=N$ where $N$ is the number of particles. These specify the motions of each $m$ th atom in the $i$ th spatial direction. The spatial extent of the different atomic motions is given in terms of mode participation ratios

$$
p=\frac{\left[\Sigma_{m} \Sigma_{i}\left(\gamma_{m, i}\right)^{2}\right]^{2}}{\Sigma_{m} \Sigma_{i}\left(\gamma_{m, i}\right)^{4}}
$$

which are calculated for motions involving boron, oxygen, or both kinds of atoms and the results are shown in Fig. 5. There, the quantity averaged over all atoms is shown alongside with the individual (unnormalized) contributions regarding oxygen and boron motions, the shape of the function dealing with cross $(\mathrm{O}-\mathrm{B})$ terms being easily inferred from

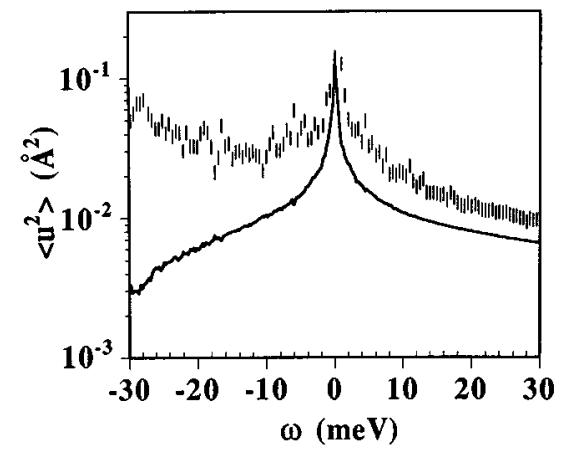

FIG. 6. Average atomic mean-square displacement (solid line) and the same quantity averaged over atoms which participate in such modes only (vertical bars).

comparison between total and the two partials. Notice that approximately $p \geqslant 0.6$ or above indicates that the modes are of extended character, showing that most of the atoms in the glass configuration participate in such motions. Two different ranges of frequencies appear as characteristic of extendedmode behavior. They correspond to motions of mainly oxygen (from some 30 to $90 \mathrm{meV}$ ) and boron atoms (from approximately 135 to $180 \mathrm{meV}$ ), being the motions of the two atomic species outside of that ranges substantially localized. As expected, the motions of B-O pairs are spatially confined for all the range of frequencies. It also seems remarkable that, contrary to other cases studied so far such as simple Lennard-Jones systems, ${ }^{17}$ the distribution of localized modes is not confined to both low- and high-frequency ends of the spectrum, but covers a rather substantial range of it.

The harmonic contribution to the $\left\langle u^{2}\right\rangle$ atomic meansquare displacements can be readily evaluated from the mode eigenvectors ${ }^{11} \gamma_{m, i}$ using

$$
\left|u_{i}^{m}\right|=\left(\frac{\hbar}{N M_{m} \omega}\right)^{1 / 2}\left|\gamma_{m, i}\right|
$$

with $M_{m}$ the mass of the $m$ th atom and it is shown in Fig. 6 . The interesting point to note regards the rather different results which are obtained whether the calculated displacements are averaged over all atoms within the sample or taken over the number of atoms participating in such modes only. The results shown in Fig. 6 indicate that whereas for real frequencies above some $20 \mathrm{meV}$ both quantities are not too far from each other, a rather dramatic difference is readily observed in the region of frequencies corresponding to unstable modes. Also notice that the absolute magnitude of such displacements has to be compared with the value of $\left\langle u^{2}\right\rangle \approx 0.06 \AA^{2}$ calculated from the average atomic displacements directly obtained from the simulation run or with that of $0.096 \AA^{2}$ calculated under the quasiharmonic approximation from the frequency distributions shown in Fig. 3. The values averaged over frequencies corresponding to the two curves shown in Fig. 6 are $1.6 \times 10^{-3} \AA^{2}$ and $3.4 \times 10^{-3}$ $\AA^{2}$, respectively, which are about one order of magnitude smaller than the total atomic displacement. The important thing to note here is that, in common with other systems explored so far, ${ }^{21,22}$ the higher-amplitude motions which correspond to the imaginary-frequency side of the spectrum only involve a reduced number of atoms. In fact as can be 
judged from a joint inspection of Figs. 5 and 6, motions with frequencies below $-20 \mathrm{meV}$ are executed by clusters of less than 50 atomic masses. It is worth pointing that such an estimate for the participation ratio is comparable albeit somewhat smaller than that of $N_{s}=68$ derived from macroscopic data given in Ref. 3 and used to parametrize the softpotential for this glass.

One of the advantages of the decomposition of the dynamics in terms of the INM regards the access to the mode eigenvectors so that the motion of the $l$ th atom within the $\lambda$ th mode is fully specified by its characteristic frequency and its $\gamma_{l}^{\lambda}$ vector displacement. Following procedures developed by Carpenter and Pelizzari, ${ }^{11}$ the one-phonon partial scattering terms are written as ${ }^{11}$

$$
\begin{aligned}
S^{1}(Q, \omega)= & \sum_{l, m} S_{l, m}^{1}(Q, \omega) \\
= & \sum_{l, m} \sum_{\lambda} \frac{\hbar^{2} \operatorname{csch}\left(\hbar \omega^{\lambda} / 2 k_{B} T\right)}{4 \hbar \omega^{\lambda}}\left[\delta\left(\omega-\omega^{\lambda}\right)\right. \\
& \left.+\delta\left(\omega+\omega^{\lambda}\right)\right] F_{l, m}^{\lambda}(Q)
\end{aligned}
$$

where $\omega^{\lambda}$ stands for the frequency of the $\lambda$ th mode where nuclei $l$ and $m$ move obeying a partial inelastic structure factor

$$
\begin{aligned}
F_{l, m}^{\lambda}(Q) / Q^{2}= & \frac{1}{\sqrt{M_{l} M_{m}}}\left[\frac{1}{3}\left(\gamma_{\mathbf{l}}^{\lambda} \cdot \gamma_{\mathbf{m}}^{\lambda *}\right) j_{0}\left(Q d_{l, m}\right)\right. \\
& +\left(\frac{1}{3}\left(\gamma_{\mathbf{l}}^{\lambda} \cdot \gamma_{\mathbf{m}}^{\lambda *}\right)-\frac{1}{d_{l, m}^{2}}\left(\mathbf{d}_{\mathbf{l}, \mathbf{m}} \cdot \gamma_{\mathbf{m}}^{\lambda *}\right)\right) \\
& \left.\times j_{2}\left(Q d_{l, m}\right)\right],
\end{aligned}
$$

where $M_{l}$ stands for the mass of atom $l$ th, which is separated by an equilibrium distance of $d_{l, m}$ from atom $m$, and $j_{0,2}$ are spherical Bessel functions. Since the $F_{l, m}^{\lambda}(Q)$ inelastic form factors can be calculated in closed form from knowledge of the $\gamma_{l}^{\lambda}$ vector displacements, a real-space correlation function can be defined as ${ }^{11,23}$

$$
D_{r}\left(r, \omega^{\lambda}\right)=\frac{2}{\pi} \int_{0}^{\infty} d Q Q \sum_{l, m} F_{l, m}^{\lambda}(Q) \sin (Q r)
$$

in analogy with the usual treatment for the static (diffraction) case. The $D_{r}(r, \omega)$ correlation function so obtained thus contains relevant geometric information regarding the identification of atom pairs executing motions at a given frequency as well as their relative phases.

Several cuts over the $D_{r}(r, \omega)$ surface are shown in Fig. 7. The selected energy transfers correspond to frequencies close to the strong low-frequency peak in the $Z(\omega)$ distributions shown in Fig. 3 (i.e., the "Boson peak"), as well as to other relatively well-defined features seen there. The first comment regarding those graphs regards the well-defined phase relationships characteristic of motions involving particles separated by less than some $4-5 \AA$. The fact that such peaks are mostly positive (or negative) serves to identify the in-phase (acoustic) or out-of-phase (optic) character of the

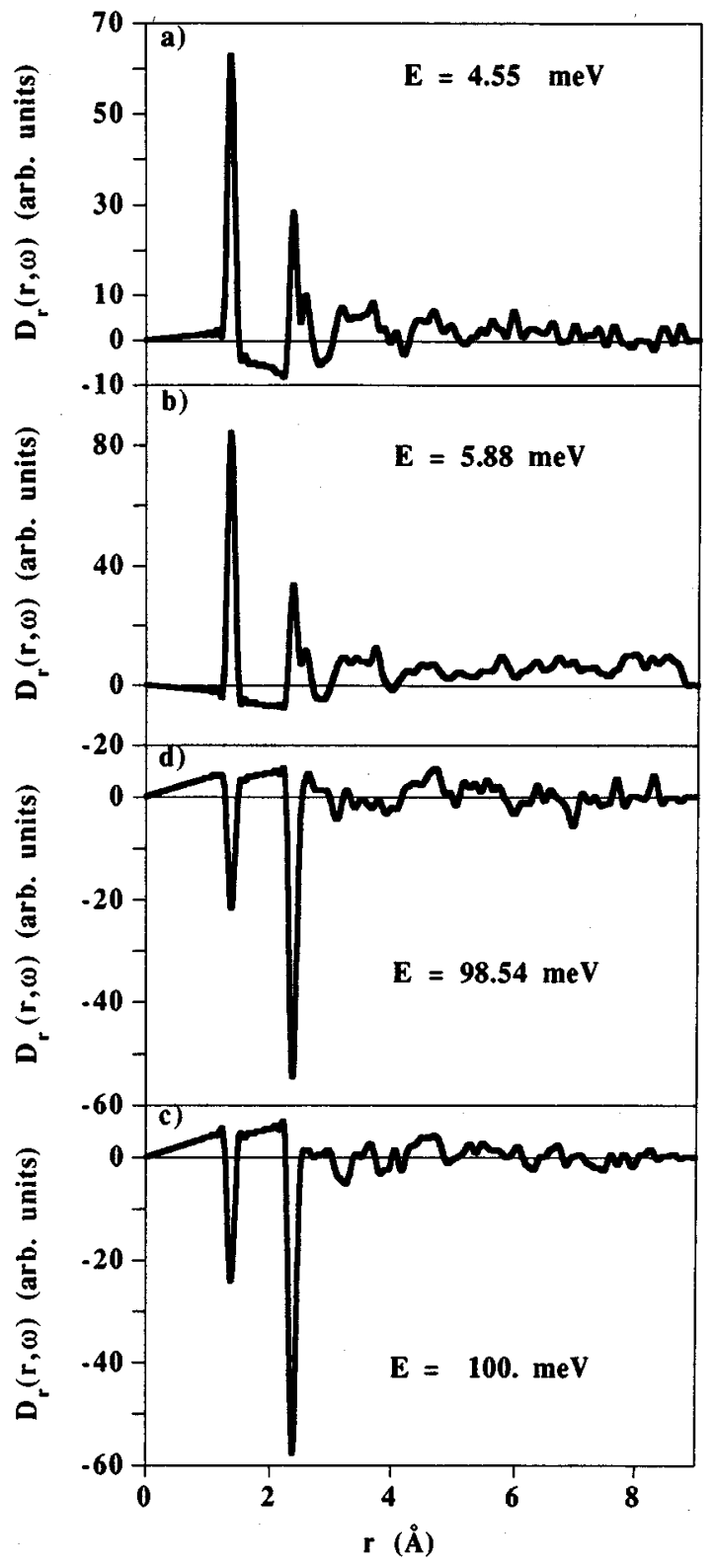

FIG. 7. Real space $D_{r}(r, \omega)$ correlation functions for different values of the energy transfers given in the inset.

modes here selected (notice that each one of the graphs corresponds to a normal mode). The $D_{r}(r, \omega)$ curve corresponding to the lowest energy $(4.55 \mathrm{meV})$ can be taken as a characteristic one for modes with energies below some $7 \mathrm{meV}$. They show rather localized motions where B-O, O-O, and B-B pairs execute in-phase movements involving up to second neighbors (i.e., involve distances up to 5-6 $\AA$ ). The acoustic character of modes within this range of frequencies is also ascertained by comparison of the relative intensities of the stronger peaks in $D_{r}(r, \omega)$, with those found in the static $T(r)$ pair-correlation function.

The three higher-lying modes at 80,90 , and $100 \mathrm{meV}$ show a strong "optical" character, also being more extended than the ones at lower energies, a fact which agrees with the participation ratios discussed above. That at $80 \mathrm{meV}$ corresponds in frequency to mode 4 of Galeener and Thorpe ${ }^{5}$ 
which was assigned to mostly B motions on the basis of comparisons between experimental Raman isotopic shifts and calculations based upon a central-force model. ${ }^{16}$ Our result agrees with such a view and shows that mainly B atoms vibrate within this mode, which has a somewhat extended character as seen by the feature at $r \approx 8 \AA$. In contrast, our result for that at $\approx 90 \mathrm{meV}$, an energy comparable to mode 5 of the referred authors, ${ }^{5}$ shows that such a mode involves both out-of-phase extended motions of B-O and O-O pairs in a similar extent whereas a limited extent of B-B pairs move in-phase. Finally it is worth remarking that the mode at 100 $\mathrm{meV}$ gives rise to an isolated peak, as shown in the inset of Fig. 3(a), which could be identified in part with that assigned to breathing motions of the boroxol ring. Since such structures are absent in this simulation its origin has to be assigned to a different entity. Notice that it corresponds to predominantly out-of-phase $\mathrm{O}-\mathrm{O}$ motions involving up to second neighbors as shown by the significant structure at approximately $5 \AA$ which basically coincides with the longest one within a boroxol ring.

\section{Collective behavior}

Up to this point, only the harmonic part of the dynamics has been studied in terms of space-dependent motions after its decomposition into individual INM's. However, as evidenced in Fig. 3, a rather substantial number of states with energies below some $15 \mathrm{meV}$ are strongly anharmonic in nature. The intensities within such a range of frequencies are known to give rise to a characteristic low-frequency inelastic peak commonly referred to as the "Boson" peak in the literature dealing with glassy dynamics. Such a feature is also visible in spectra measured at $Q \rightarrow 0$ (i.e., the Brillouin zone center if the material were a powder) by Raman scattering. This has led some to infer that such a spectral feature does not exhibit any substantial dependence with the wave vector. If this were the case, then the single-particle character of the excess modes would then be ensured. On the contrary, if some nontrivial spatial dependence is found for these intensities, then the origin of such low-energy excitation should be sought in terms of many-particle correlations. Because of the limitations of the neutron kinematics and the relatively low values of the momentum transfers required to reach full hydrodynamic behavior, no unambiguous experimental evidence regarding this particular has ever been produced. Such a difficulty can be circumvented by simulational means employing large sets of particles, and therefore we set our efforts towards the analysis of the wave-vector dependence of the simulated total (i.e., anharmonic effects included) $S(Q, \omega)$.

Figure 8 shows calculated spectra spanning from the lowest reachable $Q$ values, up to $Q \approx 1.345 \AA^{-1}$, that is well within the experimentally accessible range. The $S(Q, \omega)$ spectrum displayed in the bottom-right frame shows a shape characteristic of those for $Q \geqslant 1 \AA^{-1}$ which can be scaled to a common one once the intensity modulation dictated by the static $S(Q)$ and those of inelastic nature which follow a law proportional to $Q^{2}$ are accounted for. Such a null (or at least rather feeble) dependence with wave vectors is in stark contrast with the one observed for wave vectors below some $0.14 \AA^{-1}$. The relatively sharp feature centered at $\approx 5 \mathrm{meV}$ with a width of about $3.5 \mathrm{meV}$ shown in $S(Q, \omega)$ for $Q=1.345 \AA^{-1}$ corresponds to the "Boson" peak of this material. It seems clear after inspecting the graph that the spectrum corresponding to $Q \approx 0.035 \AA^{-1}$ shows vanishing intensity at frequencies comparable with those of the "Boson" peak, and only at $Q \approx 0.1 \AA^{-1}$ and above the intensity in such a frequency region starts to build up. In consequence, the feature sampled by Raman scattering (i.e., characteristic wave vectors of the order of $10^{-3} \AA^{-1}$ ) within a similar range of frequencies has to correspond to either second or higher-order processes involving wave vectors almost equal in magnitude and directed oppositely, or to zone-center scattering of the same kind of that enabling the detection of acoustic phonons in polycrystals within the $\mathrm{THz}$ range.

To explore with somewhat greater detail the wave-vector dependence of the spectra, a comparison between the $S(Q, \omega)$ structure factors and those expressed as $J_{L}(Q, \omega)=\omega^{2} / Q^{2} S(Q, \omega)$ is also provided in Fig. 8. The rationale behind such an exercise stems from the fact that for a sharp (hydrodynamic) phonon, that is a well-resolved excitation of sonic origin, the corresponding $J_{L}(Q, \omega)$ would be a narrow function of frequency with maxima at $\omega_{\max }=\sqrt{\omega_{b}^{2}+\Gamma^{2}}$, given in terms of a "bare" frequency $\omega_{b}$ dressed by the $\Gamma$ damping term. Within such a (low- $Q$ ) limit, most of the spectral power is attributable to sound-wave-like excitations, and therefore $\omega_{\max }$ can be rightly interpreted as the frequency of a longitudinal sound wave. If this were the case, then the $\omega_{\max }$ frequency derived from maxima in $J_{L}(Q, \omega)$ should become close to that defined from the square root of the reduced second frequency moment of the $S(Q, \omega)$, that is,

$$
\begin{gathered}
\omega_{0}=\left[\int d \omega \omega^{2} S(Q, \omega) / S(Q)\right]^{1 / 2}, \\
\lim _{Q \rightarrow 0} \omega_{0}=c_{T} Q,
\end{gathered}
$$

where $c_{T}=\sqrt{\gamma} c_{0}$ stands for the isothermal sound speed, which is given in terms of the $\gamma$ ratio of specific heats and the adiabatic sound velocity. ${ }^{24}$ The calculated values for $\omega_{0}$ and $\omega_{\max }$ are shown in Fig. 9 alongside with the hydrodynamic sound dispersion laws for the $v_{l}$ longitudinal, $v_{t}$ transverse, and average velocity $v_{\text {eff }}=\left(3 /\left[1 / v_{l}^{3}+2 / v_{t}^{3}\right]\right)^{1 / 3}=2093$ $\mathrm{m} \mathrm{s}^{-1}$, which was calculated using values of $v_{l}=3468 \mathrm{~m}$ $\mathrm{s}^{-1}, \quad v_{t}=1875 \mathrm{~m} \mathrm{~s}^{-1}$ derived from light-scattering measurements ${ }^{25}$ for $T=300 \mathrm{~K}$. The $\omega_{0}$ frequencies show the characteristic shape of a phonon dispersion curve in an isotropic medium, evidencing a shallow minimum at wavevectors close to $Q_{p}$ [i.e., that corresponding to the first maximum in $S(Q)$ ], a maximum at the "effective zone center" $Q_{p} / 2$, and an approach towards the hydrodynamic regime which follows a rather complex behavior. Above $Q_{p}$ a strong rise due to the increasing importance of higher-lying optical excitations is found. As shown in the inset, at wave vectors below some $0.08 \AA^{-1}$, that is for characteristic distances above approximately $80 \AA$, the calculated $\omega_{0}$ follows a linear dispersion $c_{T} Q=v_{\text {eff }} \sqrt{\gamma} Q$. From there up to $0.25 \AA^{-1}$ the $\omega_{0}$ frequencies approach the limit given by the longitudinal sound velocity, and from $0.3 \AA^{-1}$ a prominent change of shape is apparent. On the other hand, the values for $\omega_{\max }$ 

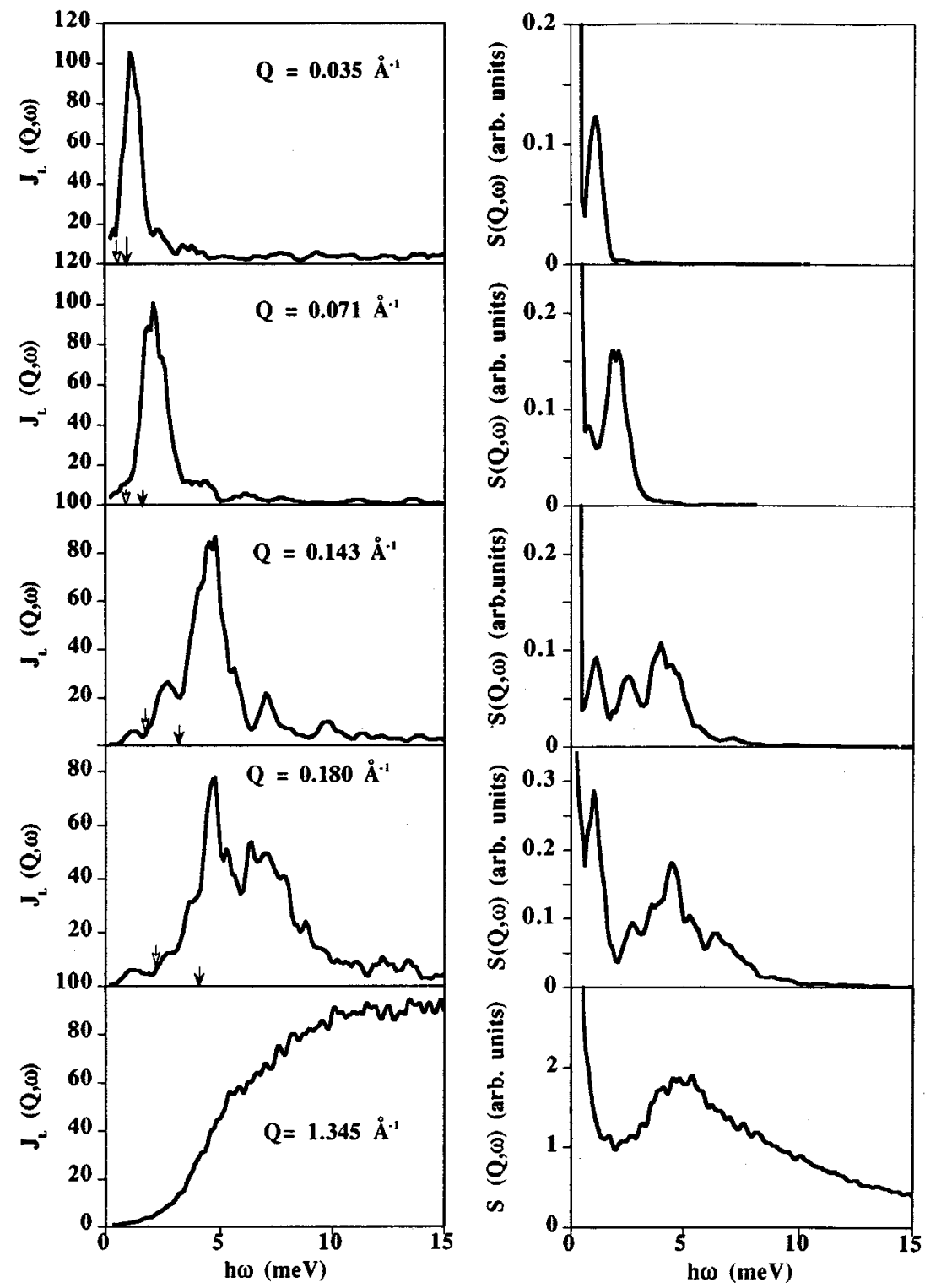

FIG. 8. The right column shows the calculated $S(Q, \omega)$ dynamic structure factors for momentum transfer values given in the insets. The column at the left-hand side shows the corresponding $J_{L}(Q, \omega)=\omega^{2} / Q^{2} S(Q, \omega)$ longitudinal current-current correlations. The arrows show the frequencies which would correspond to hydrodynamic excitations with frequencies $v_{l} Q$ and $v_{t} Q$ corresponding to the propagation of longitudinal and transverse sound.

display a far stronger dispersion which if analyzed in terms of phase velocities would correspond to ones well above hydrodynamic sound. In fact, as shown in Fig. 9(b), the phase velocities $v_{p}=\omega / Q$ associated with $\omega_{\max }$ are about 1.4 times that of longitudinal hydrodynamic sound, whereas those corresponding to $\omega_{0}$ lead to the correct hydrodynamic limit. The origin of such a discrepancy arises from the contribution to the $J_{L}(Q, \omega)$ current-current spectra of inelastic intensities corresponding to the lowest excitations of "optical" origin which are barely visible in $S(Q, \omega)$ but are greatly enhanced in $J_{L}(Q, \omega)$ due to the $\omega^{2}$ factor (for a discussion of these matters within the context of fast-sound modes in liquids see (Ref. 26). In consequence, only the $\omega_{0}$ 's can be rightly interpreted as physical frequencies identifiable in the hydrodynamic limit with those characteristic of sound propagation.
However, the comparison of $\omega_{0}$ and $\omega_{\max }$ serves to give some estimation about the onset of full hydrodynamic behavior, where both dispersion branches should merge. As seen from the inset of Fig. 9(a), such a regime has not yet been attained even at the lowest explored wave vector. An estimate of the range of wave vectors where such a crossover takes place can be made after extrapolation at $Q \rightarrow 0$ of $\omega_{\max }(Q)$, subtraction of such a constant and location of the point where it now joins the extrapolated $\omega_{0} \rightarrow 0$ line [the finite curvature of $\omega_{\max }(Q)$ is clearly seen after converting the frequencies to phase velocities, see Fig. 9(b)]. This yields a value of $Q \approx 0.02 \AA^{-1}$ corresponding to distances of some $300 \AA$, a figure to be compared with the one of $105 \AA$ $\left(Q \approx 0.06 \AA^{-1}\right.$ ) recently found by us in a study on a densegas mixture. $^{26}$ 

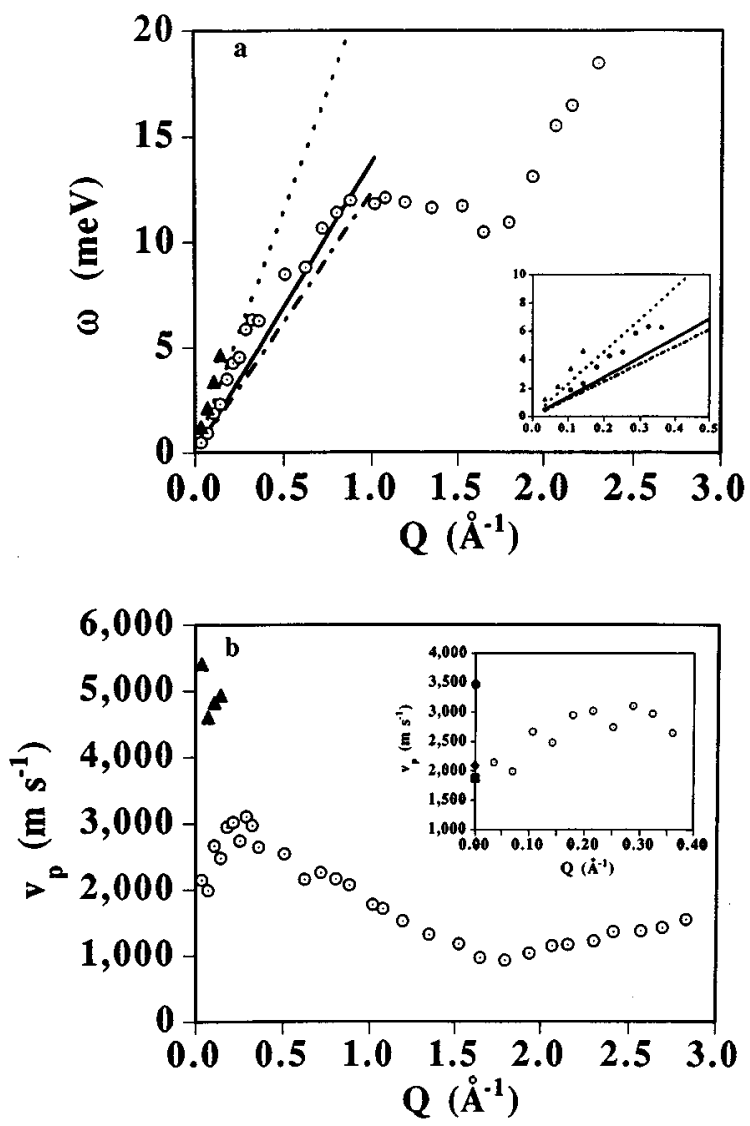

FIG. 9. (a) Wave-vector dependence of the $\omega_{0}$, square root of the normalized second frequency moments of $S(Q, \omega)$ (circles with a dot), $\omega_{\max }$, corresponding to maxima in $J_{L}(Q, \omega)$ (filled triangles). The straight lines represent hydrodynamic dispersion laws for the $v_{l}$ longitudinal sound velocity (dashes), $v_{t}$ transverse sound velocity (dash-dots), and $v_{\text {eff }}$ average velocity (solid line). The inset shows an enlargement of the low- $Q$ region. (b) Phase velocities as derived from $\omega_{0} / Q$ (circles with a dot) and $\omega_{\max } / Q$ (triangles). The inset shows an enlargement on the low- $Q$ region showing the limiting values for $v_{l}$ (filled circle), $v_{t}$ (filled square), and $v_{\text {eff }}$ (filled lozenge).

\section{CONCLUSIONS}

Although the model potential has to be improved to be fully compatible with some experimental observations (i.e., the presence of large amounts of boroxol rings ${ }^{8}$ ), the present calculations can be regarded as reliable since for the relatively low temperatures considered here [i.e., with respect to the full frequency extent of $Z(\omega)$ ], a more detailed description of the higher-frequency (above some $30 \mathrm{meV}$ ) dynamics does not seem to be essential as far as one is only concerned with properties defined in terms of integrals over the frequency distributions.

The results provided here show that far from being of single-particle character the excess modes evidence a strong collective nature which is only manifested at rather large spatial scales. This has immediate implications regarding the possible relationship between the presence of the "Boson" peak and medium-range order in glasses, a rather controversial topic at present. ${ }^{27}$ Our results show that spectral intensities corresponding to such a peak only develop for wave vectors above some $Q \approx 0.1 \AA^{-1}$, which corresponds to a scale of some $60 \AA$. Such a length is about three to six times larger than those discussed within the context of approaches ${ }^{27}$ which try to relate the frequency of the maximum of the "Boson" peak $\omega_{B}$ with a characteristic length $l$ below which localized long-wavelength, heat-carrying phonons are localized by means of some strong-scattering mechanism. In the simplest of those treatments, $\omega_{B}=2 \pi v A / l$ where $v$ is the sound velocity and $A$ a constant close to 1 (i.e., $A=1$ for a Debye model). It seems clear then that the characteristic frequency of $\omega_{B} \approx 5 \mathrm{meV}$ cannot be reconciled with such a simple model since substitution of the relevant values for the constants will give a value for such a frequency about five times shorter than the observed one.

The present results are also at odds with attempts ${ }^{28}$ to relate such characteristic length with the position in $Q$, $Q_{p}$, or the width $\Delta Q_{p}$ of the first diffraction peak of $S(Q)$. The lengths associated with the experimental values of those quantities are of 4 and $9.2 \AA$ and those from simulation give 4.6 and $5.7 \AA$, respectively. In both cases such distances are far shorter than that discussed above and, on the other hand, no distinctive feature appears in the simulated $S(Q, \omega)$ for the respective values of the wave vector.

Finally, our findings can also be rationalized within the "soft potential" model ${ }^{3}$ since, as discussed above, the largest displacements' amplitudes are those of unstable modes arising from strongly strained configurations.

\section{ACKNOWLEDGMENTS}

This work has been supported in part by DGICYT Grant No. PB92-0114-C04. Extensive discussions with Dr. A.C. Wright and Dr. A.C. Hannon as well as the sharing of their experimental data are kindly acknowledged. Dr. J. Dawidowski is acknowledged for a critical reading of the manuscript.
${ }^{1}$ U. Buchenau, C. Schönfeld, D. Richter, T. Kanaya, K. Kaji, and R. Wehrman, Phys. Rev. Lett. 73, 2344 (1994), and references therein.

${ }^{2}$ J. Colmenero, A. Arbe, and A. Alegria, Phys. Rev. Lett. 71, 2603 (1993).

${ }^{3}$ U. Buchenau, Yu.M. Galperin, V.L. Gurevich, and H.R. Schober, Phys. Rev. B 43, 5039 (1991); U. Buchenau, Yu.M. Galperin, V.L. Gurevich, D.A. Parshin, M.A. Ramos, and H.R. Schober, ibid. 46, 2798 (1992), and references therein.

${ }^{4}$ F.J. Bermejo, A. Criado, and J.L Martinez, Phys. Lett. A 195, 236 (1994).

${ }^{5}$ R.J. Bell, A. Carnevale, C.R. Kurkijan, and G.E. Peterson, J. NonCryst. Solids 35-36, 1185 (1980); Philos. Mag. B 43, 389 (1981); F.L. Galeener and M.F. Thorpe, Phys. Rev. B 28, 5802 (1983); R.A. Barrio, F.L. Castillo-Alvarado, and F.L. Galeener, ibid. 44, 7313 (1991), and references therein. A recent quantum- 
chemical calculation of structural and vibrational properties or boroxol ring sites is given by J.A. Tosell, J. Non-Cryst. Solids 183, 307 (1995).

${ }^{6}$ A.C. Hannon, R.N. Sinclair, and A.C. Wright, Physica A 201, 375 (1993)

${ }^{7}$ A.H. Verhoef and H.V. den Hartog, J. Non-Cryst. Solids 146, 267 (1992).

${ }^{8}$ A.C. Hannon, D.I. Grimley, R.A. Hulme, A.C. Wright, and R.N. Sinclair, J. Non-Cryst. Solids 177, 299 (1994), and references therein.

${ }^{9}$ Low-temperature specific-heat data were taken from M.A. Ramos, R. Villar, S. Vieira, and J.M. Calleja, in Basic Features of the Glassy State, edited by J. Colmenero and A. Alegria (World Scientific, Singapore, 1990), p. 514; G.K. White, J. Collocott, and J.S. Cook, Phys. Rev. B 29, 4778 (1984). Highertemperature data taken from S.B. Thomas and G.S. Parks, J. Phys. Chem. 35, 2031 (1931).

${ }^{10}$ P.A.V. Johnson, A.C. Wright, and R.N. Sinclair, J. Non-Cryst. Solids 50, 281 (1982).

${ }^{11}$ J.M. Carpenter and C.A. Pelizzari, Phys. Rev. B 12, 2391 (1975).

${ }^{12}$ A. Rahman, M.J. Mandell, and J.P. McTague, J. Chem. Phys. 64, 1564 (1976); Yi Wan and R.M. Stratt, ibid. 100, 5123 (1994); R. Vallauri and F.J. Bermejo, Phys. Rev. E 51, 2654 (1995); Bembenek et al. (Ref. 17).

${ }^{13}$ M. Cho, G.R. Fleming, S. Saito, I. Ohmine, and R.M. Stratt, J. Chem. Phys. 100, 6672 (1994).

${ }^{14}$ B. Madan and T. Keyes, J. Chem. Phys. 98, 3342 (1993); 102, 78 (1995); G.J. Vijayadamodar and A. Nitzan, ibid. 103, 2169 (1995).

${ }^{15}$ H. Tanaka and I. Ohmine, J. Chem. Phys. 93, 8183 (1995).

${ }^{16}$ A.C. Hannon, A.C. Wright, J.A. Blackman, and R.N. Sinclair, J. Non-Cryst. Solids 182, 78 (1995).

${ }^{17}$ A comparison of results on a simple Lennard-Jones system calculated by energy minimization and by means of the INM approach can be gauged from H.R. Schober and B.R. Laird, Phys.
Rev. B 44, 6746 (1991) and S.D. Bembenek and B.R. Laird, Phys. Rev. Lett. 74, 936 (1995), respectively.

${ }^{18}$ A.C. Hannon (private communication).

${ }^{19}$ R.B. Stephens, Phys. Rev. B 13, 852 (1976).

${ }^{20}$ J.C.K. Hui and P.B. Allen, J. Phys. C 8, 2923 (1975).

${ }^{21}$ W. Jin, P. Vashista, R.J. Kalia, and J.P. Rino, Phys. Rev. B 48, 9359 (1993).

${ }^{22}$ C. Oligschleger and H.R. Schober, Physica A 201, 391 (1993).

${ }^{23}$ Application of this procedure to derive geometric information directly from experimental data has been reported by A.C. Hannon, M. Arai, and R.G. Delaplane, Nucl. Instrum. Methods A354, 96 (1995), and references therein. See also, F.J. Bermejo, M. Garcia-Hernandez, T. Mason, J.L. Martinez, E. Enciso, and A. Criado, Phys. Rev. B 50, 13286 (1994), regarding its usefulness for the analysis of experimental data on glassy Se.

${ }^{24}$ Ultrasonic data taken from P. Macedo and T.A. Litovitz, Phys. Chem. Glasses 6, 69 (1965); N. Goldblatt, R. Figgins, C.J. Montrose, and P. Macedo, ibid. 12, 15 (1971), and references therein.

${ }^{25}$ M. Grimsditch, R. Bhadra, and L.M. Torell, Phys. Rev. Lett. 62, 2616 (1989).

${ }^{26}$ For a study on the transition to hydrodynamics of a dense gas mixture see E. Enciso, N.G. Almarza, P. Dominguez, M.A. Gonzalez, and F.J. Bermejo, Phys. Rev. Lett. 74, 4233 (1995). Some discussions regarding the meaning of $\omega_{\max }$ and $\omega_{0}$ in the context of sound propagation in liquids are given in F.J. Bermejo, M. Alvarez, S.M. Bennington, and R. Vallauri, Phys. Rev. E 51, 2250 (1995); A. Criado, F.J. Bermejo, M. GarciaHernandez, and J.L. Martinez, Phys. Rev. E 47, 3516 (1993); J. Alonso, F.J. Bermejo, M. Garcia-Hernandez, J.L. Martinez, W.S. Howells, and A. Criado, J. Chem. Phys. 96, 7696 (1992).

${ }^{27}$ See C. Levelut et al., Phys. Rev. B 51, 8606 (1995), and references therein.

${ }^{28}$ S.R. Elliot, Europhys. Lett. 19, 201 (1992); V.K. Novikov and A.P. Sokolov, Solid State Commun. 77, 243 (1991); L. Börjesson, A.K. Hassan, J. Swenson, L.M. Torell, and A. Fontana, Phys. Rev. Lett. 70, 1275 (1993). 